\title{
Histological Outcome of Fuzheng Huayu plus Entecavir Combination Therapy in Chronic Hepatitis B Patients with Significant Liver Fibrosis
}

\author{
Hong-Lian Gui ${ }^{\# 1}$, Chang-Qing Zhao ${ }^{\# 2}$, Yan Wang ${ }^{3}$, Hong-Tu Gu², Wei-Jing Wang ${ }^{1,4}$, Wei Cai ${ }^{1}$, \\ Qing Guo ${ }^{1}$, Shi-San Bao ${ }^{5}$, Lie-Ming Xu*2 and Qing Xie*1 \\ ${ }^{1}$ Department of Infectious Diseases, Ruijin Hospital, Shanghai Jiao Tong University School of Medicine, Shanghai, China; \\ ${ }^{2}$ Department of Liver Cirrhosis, Shuguang Hospital, Shanghai University of Traditional Chinese Medicine, Shanghai, China; \\ ${ }^{3}$ Department of Infectious Diseases, Nanfang Hospital, Southern Medical University, Guangzhou, Guangdong, China; \\ ${ }^{4}$ Department of Infectious Diseases, Ruijin North Hospital, Shanghai Jiao Tong University School of Medicine, Shanghai, China; \\ ${ }^{5}$ Discipline of Pathology, School of Medicine Sciences and Bosch Institute, Charles Perkin Centre, University of Sydney, New South \\ Wales, Australia
}

\begin{abstract}
Background and Aims: To evaluate the efficacy of Fuzheng Huayu (FZHY), a Chinese herbal formula, plus entecavir (ETV) in regression of liver fibrosis in chronic hepatitis $B(\mathrm{CHB})$ patients with significant fibrosis/cirrhosis. Methods: The current study was a two-center, randomized, double-blind and placebo-controlled pilot study. Fifty-two currently untreated chronic hepatitis $B$ patients with Ishak fibrosis score $\geq 3$ points were identified and $1: 1$ randomized into FZHY plus ETV combination and placebo plus ETV groups. The second liver biopsy was performed after 48-week treatment. Necroinflammatory improvement and regression of fibrosis were assessed. Fine changes in different collagen features in paired liver biopsies were evaluated by dual-photon microscopy for both groups. Results: Forty-nine patients completed the full course of treatment; forty-six of them underwent second liver biopsy (for which twenty-two were in the combination group and twenty-four were in the control group). Compared to those in the control group, patients in the combination group had significantly higher rate of fibrosis regression ( $82 \%$ vs. $54 \%$ ) $(p<0.05)$. Furthermore, the necroinflammatory improvement was greater in the combination group than in the control group ( $59 \%$ vs. $25 \%, p<0.05)$. Among the more than 80 collagen parameters in the dual-photon analysis, 5 decreased significantly in the combination group compared to the control group $(p<0.05)$. However, no significant improvement was detected in either biochemical, virologic or serologic
\end{abstract}

Keywords: Chronic hepatitis B; Fibrosis; Fuzheng Huayu tablet; Entecavir. Abbreviations: ALT, alanine aminotransferase; AST, aspartate transaminase; BMI, body mass index; CHB, chronic hepatitis B; ETV, entecavir; FZHY, Fuzheng Huayu; HAI, histological activity index; $\mathrm{HBeAg}$, hepatitis $\mathrm{B}$ e antigen; $\mathrm{HBsAg}$, hepatitis B surface antigen; HBV, hepatitis B virus; HCC, hepatocellular carcinoma; IQR, interquartile range; LSM, liver stiffness measurement; PPS, per protocol set; RUCAM, Roussel Uclaf Causality Assessment Method; TCM, traditional Chinese medicine; ULN, upper limit of normal.

Received: 19 January 2020; Revised: 2 May 2020; Accepted: 30 June 2020

\#These authors contributed equally to this work.

*Correspondence to: Qing Xie, Department of Infectious Diseases, Ruijin Hospital, Shanghai Jiao Tong University School of Medicine, Shanghai 200025, China. Tel/Fax: +86-21-64454930, E-mail: xieqingrih@163.com; Lie-ming Xu, Department of Liver Cirrhosis, Shuguang Hospital, Shanghai University of Traditional Chinese Medicine, Shanghai 201203, China. Tel/Fax: +86-21-20156520, E-mail: xulieming@shutcm.edu.cn responses between these two groups at week 48. Conclusions: The combination therapy of FZHY plus ETV for 48 weeks resulted in a higher rate of necroinflammatory improvement and fibrosis regression than ETV alone in chronic hepatitis $B$ patients with significant fibrosis/cirrhosis. The clinical trial number is ChiCTR-TRC- 11001377.

Citation of this article: Gui HL, Zhao CQ, Wang Y, Gu HT, Wang WJ, Cai W, et al. Histological outcome of Fuzheng Huayu plus entecavir combination therapy in chronic hepatitis $\mathrm{B}$ patients with significant liver fibrosis. J Clin Transl Hepatol 2020;8(3):277-284. doi: 10.14218/JCTH.2020.00004.

\section{Introduction}

Liver fibrosis is a common pathological consequence of the long clinical course of all chronic liver disease. ${ }^{1}$ Hepatitis $B$ virus (HBV) infection is still the leading cause of liver cirrhosis and hepatocellular carcinoma (HCC) in China, despite the remarkable progress of HBV treatment that has been made in recent years. The effective inhibition of HBV replication by nucleos(t)ide analogues, e.g. entecavir (ETV), can partially regress or reverse liver fibrosis in chronic hepatitis $B(\mathrm{CHB})$ patients, thereby preventing cirrhosis and subsequent hepatic decompensation and $\mathrm{HCC}^{2}$ Although serum HBV DNA is undetectable following long-term antiviral treatment, the disease existence/progression in some $\mathrm{CHB}$ patients remains. ${ }^{3}$ Thus, histopathological outcome remains an important aspect of efficacy evaluation in CHB treatment, and contributes to clinical decision-making in terms of treatment duration and choice of medication. The current therapeutic approach in refractory liver fibrosis is to apply anti-fibrotic agents to inhibit hepatic extracellular matrix synthesis and stellate cell activation, especially in those CHB patients with significant fibrosis. ${ }^{4}$

Traditional Chinese medicine (TCM) plays an important role in developing an effective antifibrotic strategy. Fuzheng Huayu (FZHY) is a Chinese Food and Drug Administrationapproved Chinese herbal formula, consisting of Radix Salvia Miltiorrhizae (Danshen), Pollen Pini (Songhuafen), Semen Persicae (Taoren), Gynostemma Pentaphyllammak (Jiaogulan), Cordyceps (Chongcao), and Fructus Schisandrae 
Chinensis (Wuweizi). ${ }^{5}$ It has been reported that FZHY enhances the degradation of collagens in the fibrotic liver with a robust good safety and efficacy profile in patients with various chronic liver diseases, ${ }^{6,7}$ including hepatitis $B$, hepatitis $\mathrm{C}$, nonalcoholic steatohepatitis, etc. This antifibrotic effect of FZHY has also been confirmed in a USA Federal Drug Administration-approved phase II trial in patients with chronic hepatitis C which was completed in $2013 .{ }^{8}$ However, there might be some bias in the previous reports, as it was not a double-blind, multi-center study with solid high-quality clinical evidence.

Thus, to address the issues mentioned above, we designed a two-center, randomized, double-blinded and placebo-controlled pilot study to evaluate the efficacy and safety of the combination of FZHY and ETV in comparison to ETV monotherapy in CHB patients with significant fibrosis or cirrhosis (Ishak $\geq 3$ ), with particular focus on the improvement of histological outcome. Our current study may provide some insight in exploring the underlying mechanism of $\mathrm{FZHY}$ in management of $\mathrm{CHB}$.

\section{Methods}

\section{Patient recruitment}

Eligible patients consented to participate in the study between April 2011 and January 2012, and were recruited and followed-up until November 2013 at Shanghai Ruijin Hospital and Shanghai Shuguang Hospital. Inclusion criteria were: (1) ages 18 to 65 years; (2) persistent serum hepatitis B surface antigen (HBsAg) for $\geq 6$ months; (3) Ishak fibrosis score of $\geq 3$ within 1 month of liver biopsy; (4) alanine aminotransferase (ALT) $<5 \times$ the upper limit of normal (ULN); and (5) for hepatitis $\mathrm{B}$ e antigen (HBeAg)-positive patients: HBV DNA $>2.0 \times 10^{4} \mathrm{IU} / \mathrm{mL}$ and for HBeAg-negative patients: HBV DNA $>2.0 \times 10^{3} \mathrm{IU} / \mathrm{mL}$. Exclusion criteria included co-infection with other hepatitis viruses or human immunodeficiency virus, autoimmune hepatitis, alcoholic liver disease, drug or toxic hepatitis, or inherited metabolic liver disease. Moreover, decompensated liver cirrhosis, liver cancer, ALT $>5$-fold ULN, total bilirubin $>2 \times U L N$, body mass index (referred to as BMI) $>30 \mathrm{~kg} / \mathrm{m}^{2}$, treatment with antiviral therapy within 6 months before screening, and antifibrosis drug within 1 month before screening were also excluded.

\section{Trial oversight and design}

The study was conducted in accordance with the ethical guidelines of the Declaration of Helsinki and International Conference on Harmonization Guidelines for Good Clinical Practice. The protocol and amendments were approved by the ethics committee of each center (Shanghai Ruijin Hospital and Shanghai Shuguang Hospital). The investigators at each center were responsible for study management and study compliance. Informed consent was obtained from each participant or legal representative. The clinical trial number was ChiCTR-TRC-11001377.

The study was an investigator-initiated, randomized, double-blind, placebo-controlled, and two-center pilot clinical trial. The study comprised a 4-week screening period, followed by a 48 -week double-blind treatment period. Computerized randomization codes were generated; blinding of patients, investigators, and study site personnel was imple- mented and maintained by the interactive web-response system. Breaking of the blinding for any reason resulted in discontinuation from study participation. Eligible subjects were randomly assigned in a 1:1 ratio to one of the following two groups: a combination group (receiving $0.5 \mathrm{mg}$ ETV once daily and $1.6 \mathrm{~g} \mathrm{FZHY} \mathrm{three} \mathrm{times} \mathrm{daily)} \mathrm{or} \mathrm{a} \mathrm{control} \mathrm{group}$ [receiving $0.5 \mathrm{mg}$ ETV once daily and $1.6 \mathrm{~g}$ placebo (similar in taste, shape and color to FZHY; main ingredients include $1 \%$ FZHY) three times daily]. Follow-up assessments were performed every 12 weeks.

The subjects were required to visit the site at $12 \mathrm{~W}, 24 \mathrm{~W}$, $36 \mathrm{~W}$ and $48 \mathrm{~W}$ during the study treatment period, and the drugs were dispensed at each visit. Drug compliance was emphasized and monitored at each visit. As a result, the rates of taking all medicines were all in the range of $80 \%$ to $100 \%$. The second liver biopsy was performed right after the 48-week treatment was completed.

\section{Clinical assessment}

All patients were monitored every 12 weeks with the following determination: HBsAg, anti-HBs, HBeAg, anti-HBe, HBV DNA, and routine hematological and biochemical tests, $\alpha$-fetoprotein, and abdominal ultrasonography. Using transient elastography (FibroScan; Echosens, Paris, France), liver stiffness measurement (LSM) was made every 24 weeks by experienced operators and according to the process previously described. ${ }^{9}$

\section{Histopathology}

All of the $52 \mathrm{CHB}$ patients underwent liver biopsy at baseline. In addition, 46 of the patients underwent a second liver biopsy at week 48. All the liver biopsies were routinely processed and sectioned at $5-\mu \mathrm{m}$ thickness for Masson trichrome staining. All of the liver biopsies were scored by two independent histopathologists in a double-blinded fashion. ${ }^{10}$ Assessment criteria were as follows: necroinflammatory activity was assessed with modified Knodell histological activity index (HAI) score ${ }^{11}$ and was classified into 5 grades, being 0-no (HAI: 0), 1-minimal (HAI: 1-3), 2-mild (HAI: 4-8), 3-moderate (HAI: 9-12), and 4-severe (HAI: 13$18)$; whereas determination of fibrosis stage was made on Masson trichrome-stained sections, using 7-stage Ishak fibrosis scoring, ${ }^{11}$ which ranged from 0 (no fibrosis) to 6 (cirrhosis).

\section{Efficacy analysis}

The prespecified primary efficacy parameter was the percentage of fibrosis regression, which was defined as $\geq 1$-stage decrease by the Ishak scoring system, from the baseline to week 48 . The prespecified secondary efficacy parameter was the percentage of necroinflammatory improvement, which was defined as $\geq 1$-grade reduction in Knodell necroinflammatory score without worsening of fibrosis from the baseline to week 48. Additional prespecified efficacy parameters were biochemical, virologic and serologic responses at week 48 .

\section{Safety analysis}

Safety was assessed by investigators at each patient visit based on vital signs, physical examinations, adverse events, 
concomitant medication assessments as well as laboratory tests.

\section{Collagen measurement by dual-photon microscopy}

To explore the underlying mechanism of FZHY in management of $\mathrm{CHB}$, all the liver samples were further analyzed with dual-photon microscopy at Southern Medical University (Guangzhou, China), according to the experimental procedures as previously established in CHB cohorts. ${ }^{12}$ The main collagen parameters relating to portal collagen (portal expansion), septal collagen (bridging fibrosis), and fibrillary collagen (fine collagen distributed in the pericellular/perisinusoidal space of Disse) were identified through image acquisition and processing, and translated into quantitative variables. A list of more than 80 collagen parameters was obtained from each liver specimen. ${ }^{12}$

\section{Statistical analysis}

SAS 9.2 software was used for statistical analysis. The majority of data was assessed using the full-analysis set. A per protocol set (PPS) analysis limited to subjects who completed the 48-week treatment and a second liver biopsy was conducted to determine the main measurement outcomes. Incidence of adverse reactions was analyzed using a safety set analysis that included all enrolled subjects.

Continuous variables were presented as means \pm standard deviations or medians (interquartile range, IQR). Comparisons between the two groups were performed using $t$-tests if values were normally distributed, or by the Wilcoxon rank sum test if the distribution was not normal. Comparisons of collagen architectural features between the pre- and posttreatments were analyzed by the Wilcoxon pair test, while comparisons of collagen architectural features between the two groups were analyzed by the Hodges-Lehmann estimate of location shift test. Frequency data were expressed as numbers and percentages, and were compared using the chi-square test or the Fisher's exact test, or the CochranMantel-Haenszel test in ranked data, as appropriate. $p$ values $\leq 0.05$ (two-sided) were statistically significant.

\section{Results}

\section{Study population}

A total of $52 \mathrm{CHB}$ patients with significant fibrosis/cirrhosis were identified and randomized either to the combination group ( $n=26)$ or to the control group $(n=26)$. One patient was eliminated due to HCC at the time of the week 24 follow-up and 2 patients dropped out of the combination group due to relocation (Fig. 1). Finally, 49 patients completed the full course of treatment and 46 underwent the second liver biopsy. Thus, 52 patients were included in the safety set analysis, 51 in the full-analysis set analysis, and 46 in the PPS analysis.

The baseline characteristics of the patients are presented in Table 1. There was no significant difference in the age, BMI, sex, HBeAg status, HBV DNA level, biochemical test results, LSM nor histological findings between the combination and the control groups.

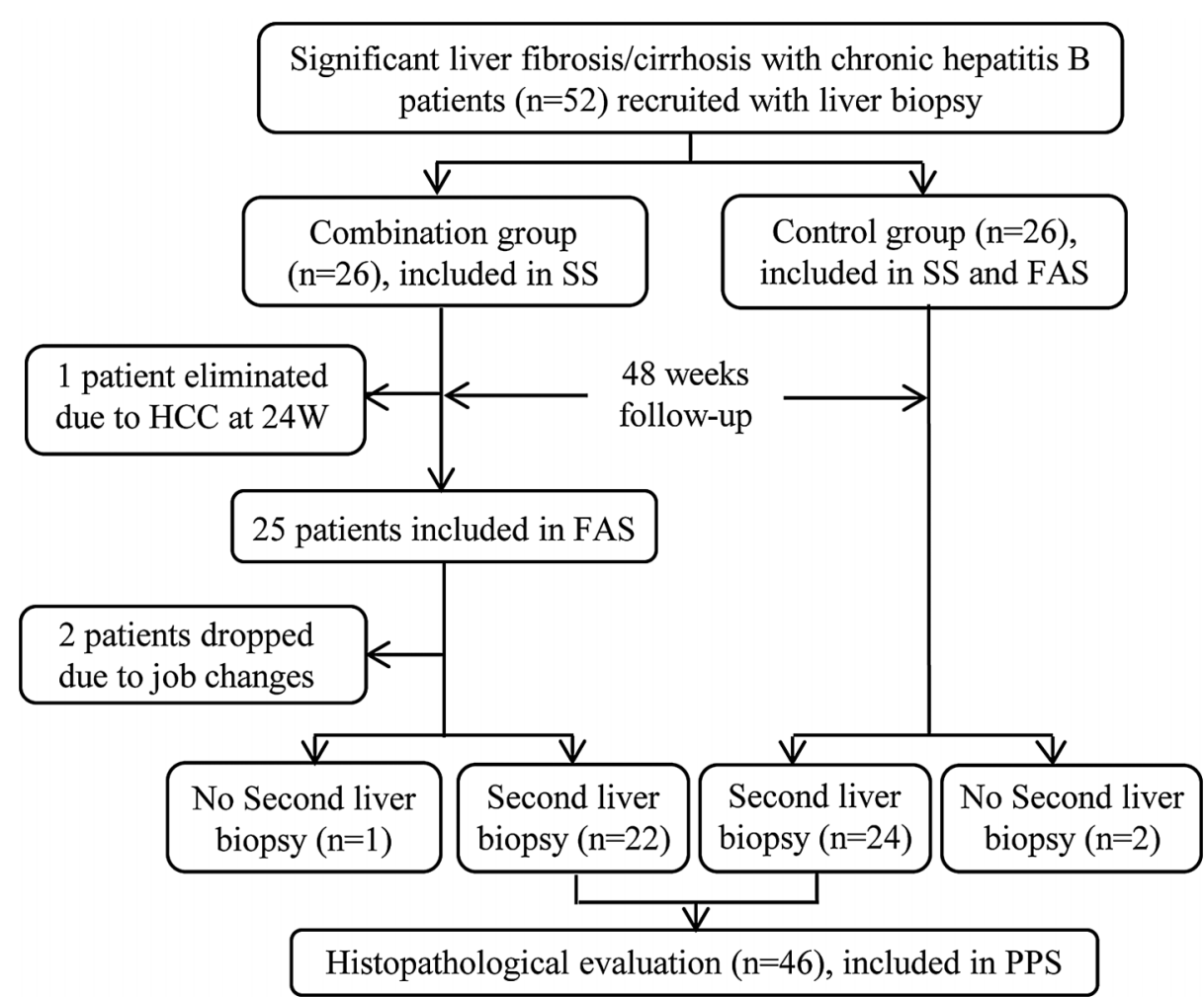

Fig. 1. Flow chart illustrating the experimental design for the patients throughout the study. 
Gui H.L. et al: FZHY and ETV in CHB with significant fibrosis

Table 1. Demographic and baseline characteristics of the patients

\begin{tabular}{|c|c|c|c|}
\hline Variables & Combination group, $n=25$ & Control group, $n=26$ & $p$-value \\
\hline Age in year & $44.48 \pm 10.31$ & $42.46 \pm 11.39$ & 0.511 \\
\hline Male gender, $n(\%)$ & $15(60.0)$ & $19(73.1)$ & 0.322 \\
\hline $\mathrm{BMI}, \mathrm{kg} / \mathrm{m}^{2}$ & $23.51 \pm 2.68$ & $22.89 \pm 2.59$ & 0.398 \\
\hline HBeAg-positive, $n$ (\%) & $15(60.0)$ & $14(53.8)$ & 0.779 \\
\hline HBVDNA, log IU/ml & $5.94 \pm 0.92$ & $5.87 \pm 1.23$ & 0.826 \\
\hline ALT, IU/L & $42.24 \pm 22.23$ & $43.35 \pm 24.93$ & 0.868 \\
\hline AST, IU/L & $34.60 \pm 16.32$ & $36.88 \pm 16.17$ & 0.618 \\
\hline $\mathrm{TB}, \mu \mathrm{mol} / \mathrm{L}$ & $18.12 \pm 5.18$ & $16.15 \pm 5.46$ & 0.193 \\
\hline Albumin, $\mathrm{g} / \mathrm{L}$ & $44.30 \pm 2.99$ & $44.12 \pm 3.72$ & 0.853 \\
\hline Modified HAI grade & & & 0.928 \\
\hline 1 -minimal, $n(\%)$ & $2(8.0)$ & $0(0.0)$ & \\
\hline 2-mild, $n(\%)$ & $11(44.0)$ & $17(65.4)$ & \\
\hline 3-moderate, $n(\%)$ & $11(44.0)$ & $7(26.9)$ & \\
\hline 4-severe, $n(\%)$ & $1(4.0)$ & $2(7.7)$ & \\
\hline Ishak stage & & & 0.854 \\
\hline $3, n(\%)$ & $8(32.0)$ & $5(19.2)$ & \\
\hline $4, n(\%)$ & $4(16.0)$ & $9(34.6)$ & \\
\hline $5, n(\%)$ & $6(24.0)$ & $8(30.8)$ & \\
\hline $6, n(\%)$ & $7(28.0)$ & $4(15.4)$ & \\
\hline LSM, kPa & $13.80 \pm 8.94$ & $16.24 \pm 13.11$ & 0.442 \\
\hline
\end{tabular}

Abbreviations: ALT, alanine aminotransferase; AST, aspartate transaminase; BMI, body mass index; TB, total bilirubin; HAI, histological activity index; LSM, liver stiffness measurement.

\section{Histological response}

Histopathological comparison was performed on the PPS. There was no significant difference in ALT, aspartate aminotransferase and HBV DNA among the $46 \mathrm{CHB}$ patients with second biopsy (in which 22 patients were in the combination group and 24 were in the control group).

In assessment of fibrosis, $31 / 46$ patients (67.4\%) had improved Ishak fibrosis stage, of at least 1 point by comparing the first and second biopsies during the whole paired study (Fig. 2). The median (IQR) reduction in Ishak fibrosis stage was 1 (1-2) point in the combination group versus 1 (0$2)$ in the control group $(p>0.05)$. However, the proportion of the CHB patients with Ishak fibrosis in the combination group with at least a 1-point improvement was significantly higher than that in the control group [81.8\% (18/22) vs. $54.2 \%$ (13/ $24), p<0.05]$. Notably, all three patients who had worsened Ishak fibrosis occurred in the control group.

To determine if there was any significant improvement of fibrosis in CHB patients with the high Ishak score in response to the combination therapy, we further stratified subjects according to their baseline fibrosis status. The proportion of $\mathrm{CHB}$ patients with improved fibrosis was higher in the baseline Ishak 3-4 score subgroup $[60.0 \%(6 / 10)$ in the combination group vs. $30.8 \%(4 / 13)$ in the control group, $p=0.077]$ and in the baseline Ishak 5-6 score subgroup [100\% (12/12) in the combination group vs. $81.8 \%(9 / 11)$ in the control group, $p=0.153]$. Although no statistical significance was found, there were trends of fibrosis improvement observed in both groups. Apparently, the combination therapy

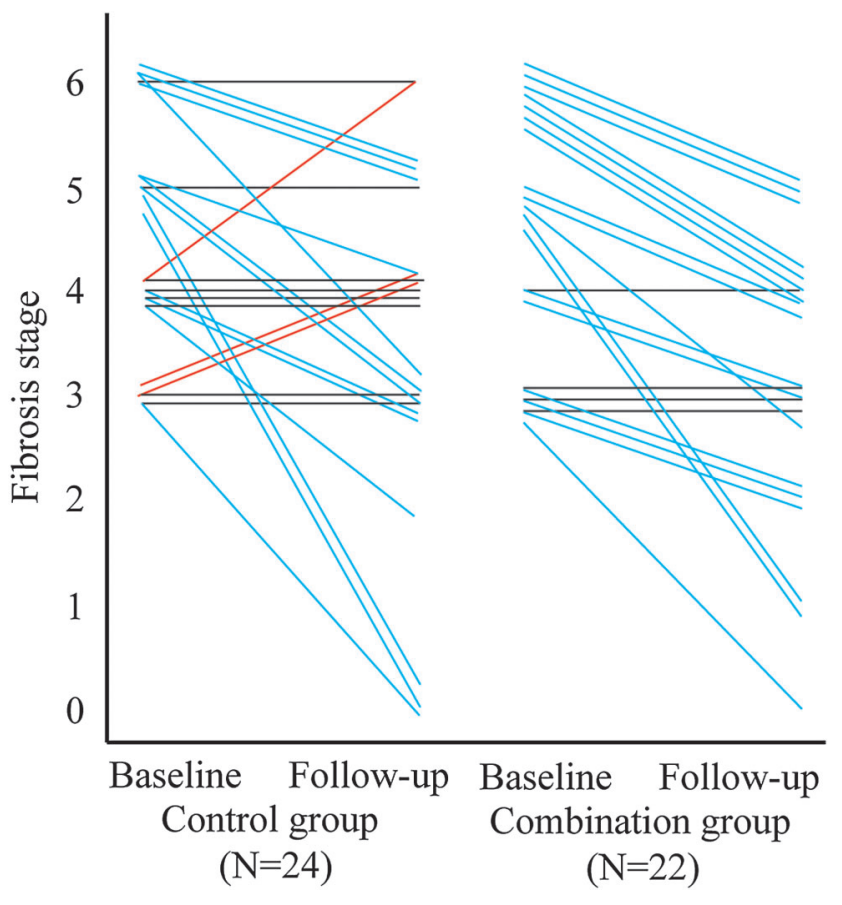

Fig. 2. Changes of Ishak fibrosis stage between the combination group and control group at baseline and at 48 weeks.

The improvement in Ishak fibrosis was significantly more common in the combination group than in the control group after 48 weeks of treatment. $p=0.021$, Cochran-Mantel-Haenszel test was used. 
performed better in each fibrosis status when compared with the control group.

The necroinflammatory activity grades from these 46 patients at the baseline and at week 48 treatment are presented in Fig. 3. Nineteen of the 46 patients $(41.3 \%)$ had improved HAI necroinflammatory grade at week $48 \mathrm{com}$ pared to the baseline biopsy. The median (IQR) reduction in the modified HAI grade from the baseline in the combination group was $1(0-1)$ point, but was $0(0-0.5)$ in the control group $(p<0.05)$. Thirteen patients $(59.1 \%)$ in the combination group had improved HAI grade of at least 1 point and no patients had worsened HAI grade, whereas only six patients (25.0\%) had improved HAI grade at least 1 point and three patients $(12.5 \%)$ had worsened HAI grade in the control group. Thus, our data demonstrated that the proportion of CHB patients with at least a 1-point improvement in HAI grade in the combination group was significantly higher than that in the control group $(p<0.05)$.

\section{Biochemical, virologic and serologic responses}

Biochemical, virologic and serologic comparisons were assessed using the full analysis set (Table 2). There was no significant difference in biochemical endpoints at week 48 between the two groups. The median (IQR) reduction of ALT levels were 16 (4-43) and 16 (2-40) IU/L in the combination group and control group, respectively, at week $48(p=0.76)$. Virologic response at week 24 was generally consistent with the response at week 48 . At week 48,91\% (21/23) and 92\% $(23 / 25)$ of CHB patients achieved complete viral response (defined as HBV DNA $<500 \mathrm{IU} / \mathrm{mL}$ ) in the combination and

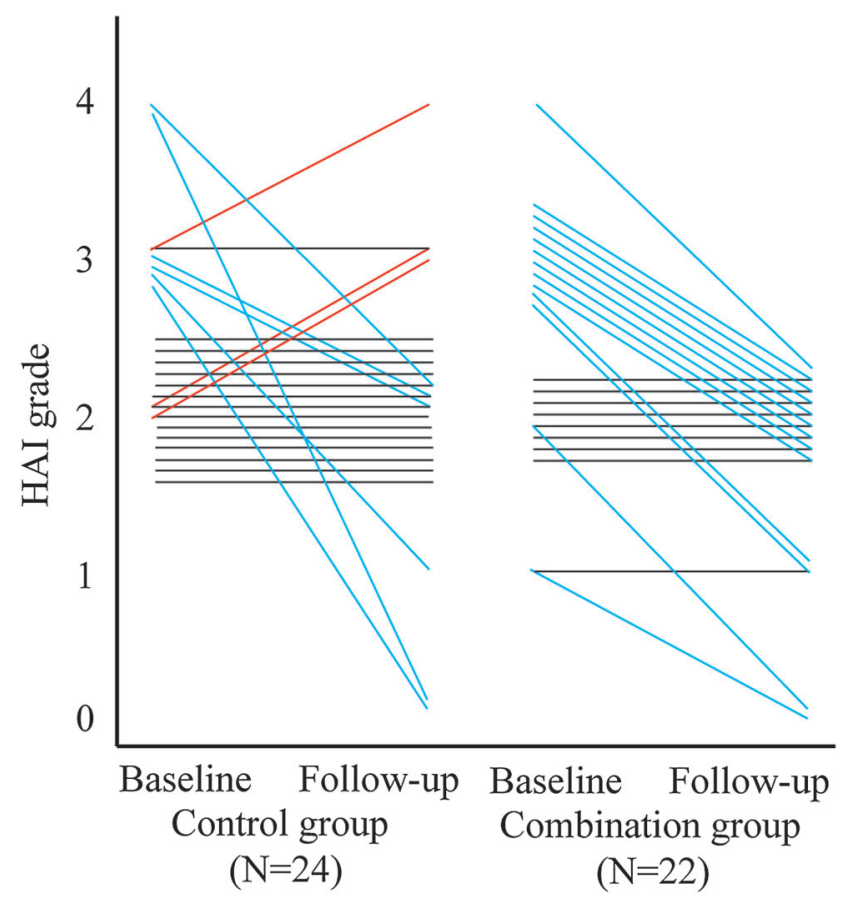

Fig. 3. Changes of HAI grade between the combination group and control group at baseline and at $\mathbf{4 8}$ weeks.

The improvement in necroinflammatory activity was significantly more common in the combination group than in the control group after 48 weeks of treatment. $p=0.009$, Cochran-Mantel-Haenszel test was used. control groups, respectively. No significant difference in $\mathrm{HBeAg}$ seroconversion was observed between the combination group $(7.7 \%, 1 / 13)$ and the control group $(28.6 \%, 4 / 14)$ at week $48(p=0.33)$.

\section{Changes in collagen parameters}

In terms of significant changes of fibrosis stages between week 48 and the baseline biopsies after FZHY and ETV combination treatment, we further evaluated the fine changes in different collagen parameters from portal, septal and fibrillary areas using dual-photon microscopy in both groups with paired liver biopsies (Table 3 ). Compared with paired liver biopsies, 17 collagen parameters were significantly improved in the combination group, while only 2 were improved in the control group.

Moreover, five collagen parameters in the combination group decreased significantly over those in the control group $(p<0.05)$, including the proportion of collagen in tissue, collagen fiber area, collagen fiber length, septal collagen fiber area, and septal collagen fiber width, supporting the importance of septa resorption in fibrosis regression. ${ }^{13}$

\section{Safety analysis}

No abnormality was observed in the routine urine and blood tests, renal function and ECG, suggesting the safety of either the combined or control treatment. There were no other noticeable adverse effects observed during the study for both groups, except that one of the patients developed HCC.

\section{Discussion}

Our current study demonstrated that FZHY and ETV 48-week combined therapy significantly improved necroinflammatory activity and fibrosis compared to ETV monotherapy, with convincing histopathological evidence. Our current study was the first randomized clinical in this research field.

It is worth mentioning that the definition of fibrosis improvement was based on objective histopathology in our study and not purely on non-invasive assessment of liver fibrosis. Recently, it has been popular to use non-invasive approaches in evaluating liver conditions/severity, including in our own research group; ${ }^{9}$ however, histopathology provides the valuable tissue level evidence in assessment of liver structure, despite an invasive approach. In most of the previous FZHY and other anti-fibrotic TCM studies, ${ }^{14,15}$ noninvasive direct serum markers were utilized, including hyaluronic acid, procollagen III protein, collagen C type IV, and laminin. It should be cautious that these serum markers actually only assess matrix turnover and are not liver-specific, being easily affected by other factors (e.g. inflammation, congestion, etc.). ${ }^{16}$ Furthermore, due to high cost for the imaging facility, limited local availability and high rate of indeterminate results $(14 \sim 33 \%$ of cases, also known as a "Gray zone"), the non-invasive approaches are still considered as a supplementary approach and not replacement of liver biopsy as the gold standard for the evaluation of staging and grading in chronic hepatitis. ${ }^{17}$

A common method for assessment of fibrosis changes is semi-quantitative scoring, such as the Ishak scoring system, which uses a valuation range from 0 to 6 . However, in the era of potent anti-HBV treatment, a 7-point semi-quantitative system is a relatively gross assessment. The Beijing 
Gui H.L. et al: FZHY and ETV in CHB with significant fibrosis

Table 2. Biochemical, virologic and serologic responses at week 24 and week 48

\begin{tabular}{|c|c|c|c|c|c|c|}
\hline \multirow[b]{2}{*}{ Variables } & \multicolumn{3}{|l|}{ Week 24} & \multicolumn{3}{|l|}{ Week 48} \\
\hline & $\begin{array}{l}\text { Combination } \\
\text { group }\end{array}$ & $\begin{array}{l}\text { Control } \\
\text { group }\end{array}$ & $p$-value & $\begin{array}{l}\text { Combination } \\
\text { group }\end{array}$ & $\begin{array}{l}\text { Control } \\
\text { group }\end{array}$ & $p$-value \\
\hline N (Miss) & 25 & 26 & & $23(2)$ & $25(1)$ & \\
\hline ALT, IU/L & $32.80 \pm 12.08$ & $37.03 \pm 15.96$ & 0.29 & $30.97 \pm 12.18$ & $33.92 \pm 20.00$ & 0.54 \\
\hline Virologic response* & $19(76.0 \%)$ & $21(80.8 \%)$ & 0.68 & $21(91.3 \%)$ & $23(92.0 \%)$ & 1.00 \\
\hline $\begin{array}{l}\text { Baseline } \mathrm{HBeAg}(+) \mathrm{N} \\
\text { (Miss) }\end{array}$ & 15 & 14 & & $13(2)$ & $14(0)$ & \\
\hline HBeAg loss & $4(26.7 \%)$ & $4(28.6 \%)$ & 1.00 & $4(30.8 \%)$ & $5(35.7 \%)$ & 1.00 \\
\hline HBeAg seroconversion & $3(20.0 \%)$ & $4(28.6 \%)$ & 0.68 & $1(7.7 \%)$ & $4(28.6 \%)$ & 0.33 \\
\hline
\end{tabular}

${ }^{*}$ Virologic response was defined as serum HBV DNA $<500 \mathrm{IU} / \mathrm{mL}$.

Abbreviations: ALT, alanine aminotransferase; $\mathrm{HBeAg}$, hepatitis $\mathrm{B}$ e antigen.

Table 3. Collagen parameters with significant changes

\begin{tabular}{|c|c|c|c|}
\hline Variables & Week 0 & Week 48 & $p$-value \\
\hline \multicolumn{4}{|c|}{ Combination group } \\
\hline Portal collagen fiber number & $238.51(176.18 \sim 405.88)$ & $207.12(101.67 \sim 303.67)$ & 0.038 \\
\hline Type I collagen fiber number & $179.83(125.31 \sim 317.71)$ & $166.13(102.02 \sim 234.70)$ & 0.021 \\
\hline Type III collagen fiber number & $48.33(34.34 \sim 83.67)$ & $39.04(22.33 \sim 55.86)$ & 0.045 \\
\hline Type IV collagen fiber number & $188.48(145.29 \sim 304.74)$ & $183.28(112.57 \sim 249.55)$ & 0.043 \\
\hline Portal distributed collagen proportionate area & $224.20(163.89 \sim 382.37)$ & $202.04(146.95 \sim 285.08)$ & 0.038 \\
\hline Portal collagen fiber curvature & $161.92(117.31 \sim 275.08)$ & $144.74(89.31 \sim 207.09)$ & 0.033 \\
\hline Percentage of type II perivascular collagen & $0.09(0.05 \sim 0.14)$ & $0.04(0.03 \sim 0.09)$ & 0.007 \\
\hline Perivascular collagen fiber total number & $212.07(135.57 \sim 297.50)$ & $137.17(75.49 \sim 199.40)$ & 0.017 \\
\hline Perivascular type I collagen fiber number & $161.05(106.26 \sim 226.50)$ & $100.82(70.67 \sim 149.00)$ & 0.009 \\
\hline Perivascular type III collagen fiber number & $43.45(26.38 \sim 57.07)$ & $26.51(20.81 \sim 34.98)$ & 0.014 \\
\hline Perivascular type IV collagen fiber number & $169.21(113.46 \sim 232.02)$ & $106.07(60.73 \sim 171.40)$ & 0.020 \\
\hline Perivascular collagen fiber length & $10143(5899 \sim 12753)$ & $7179(4306 \sim 11601)$ & 0.048 \\
\hline Perivascular type I collagen fiber total number & $206.53(131.94 \sim 284.00)$ & $141.46(94.54 \sim 216.00)$ & 0.026 \\
\hline Perivascular I-I collagen fiber number & $156.24(102.99 \sim 213.75)$ & $100.23(70.00 \sim 145.20)$ & 0.017 \\
\hline Perivascular I-III collagen fiber number & $41.71(25.18 \sim 55.90)$ & $25.63(18.64 \sim 33.50)$ & 0.025 \\
\hline Perivascular I-IV collagen fiber number & $167.36(113.26 \sim 226.33)$ & $105.99(59.83 \sim 169.20)$ & 0.030 \\
\hline Perivascular collagen cross-link density & $5315(3351 \sim 8643)$ & $3183(1856 \sim 5559)$ & 0.040 \\
\hline \multicolumn{4}{|c|}{ Control group } \\
\hline Percentage of perivascular collagen & $1.26(0.84 \sim 1.98)$ & $0.56(0.42 \sim 1.27)$ & 0.013 \\
\hline Percentage of type I perivascular collagen & $1.19(0.77 \sim 1.83)$ & $0.51(0.36 \sim 1.22)$ & 0.015 \\
\hline
\end{tabular}

Classification is a new staging system, providing more accurate and improved quantification of fibrosis, which could be useful in the era of antiviral therapy assessment. ${ }^{3}$ However, The Beijing Classification is still not as perfect as the structure-based full quantitative method ${ }^{18}$ - quantitative collagen parameter measurement by dual-photon microscopy, which is the computerized quantification for staging liver fibrosis and thus may thereby reduce variability of inter-/intra-observer bias. Therefore, assessment of quantitative collagen parameters is supposed to be more sensitive in measuring liver fibrosis remodeling. The findings of quantitative collagen parameter measurement are in line with our histologic findings, which shows that the changes in collagen parameters were objective and reliable in our combination and control groups. In addition, our data strongly suggest that FZHY therapy improves histological outcome by regulating hepatic extracellular matrix metabolism during a relatively short period of anti-fibrotic therapy.

Notably, three of our patients who showed worsened Ishak fibrosis all occurred in the control group in PPS. In our study, all patients in both groups had good medication compliance. It is worth mentioning here that, for uncontrolled metabolic diseases, medication compliance should be strongly emphasized since anti-diabetic medications, such as metformin, and 
anti-lipid medications, such as statins, can both improve fibrosis. ${ }^{19}$ In our study, only one subject with type-2 diabetes was treated with insulin in the control arm and they achieved fibrosis regression at the 48-week time point, while none was treated with statins. Thus, there were no significant confounding factors in the baseline medication profile. In addition, ETV is a potent antiviral drug with a high resistance barrier for and none developed drug resistance within the 48-week therapy in our study.

As expected, there was no significant difference between FZHY plus ETV and ETV only groups for improving the biochemical, virologic and serologic responses at week 24 and week 48 in the present study. However, a recent metaanalysis demonstrated that FZHY appears to be more efficient in improving liver function index (ALT, AST and total bilirubin) and the HBV DNA-negative conversion rate compared to conventional treatment. ${ }^{6}$ The discrepancy between our current study and the meta-analysis may be due to the fact that our study is so far the only double-blind, randomized and placebo-controlled study in this field. We believe that our data are more reliable because it is well known that ETV is the major contributor to the biochemical, virologic, and serologic responses produced. ${ }^{20,21}$

Suspected cases of herb-induced liver injury caused by TCMs have been highlighted in many publications. ${ }^{22,23}$ TCMrelated liver injury is a newly emerged topic based on a causality grading by the Roussel Uclaf Causality Assessment Method (commonly referred to as RUCAM). Referencing RUCAM-based cases, none of the six herbals in FZHY is potentially hepatotoxic. In addition, FZHY was approved by the Chinese Food and Drug Administration in 2002 and launched on the Chinese market nearly 20 years ago. The quality inspection and product standardization of FZHY is well-established and strictly enforced. The content of major ingredients was measured on-site during the manufacturing processes, and as a quality inspection standard; as such, the procedures can be fully reproducible. ${ }^{7}$ The fact that the active components of FZHY formula and their potential mechanism in inhibiting hepatic stellate cells' viability have been identified using network pharmacology and transcriptomics ${ }^{24}$ has provided the evidence underlying its known antifibrotic action.

Our study has several limitations. Firstly, the trial was not long enough to assess the effect of FZHY on clinical outcomes, such as HCC, decompensated cirrhosis, liver transplantation and death. Secondly, we tried to employ some noninvasive fibrosis models at baseline and at 24-week of treatment to explore possible predictors for histological response. However, no specific predictors of response were identified in this study, probably due to the relatively small sample size. Thirdly, since a persistent low level of residual HBV may promote fibrosis progression during therapy, ${ }^{25}$ a lower limit of viral load (e.g., $20 \mathrm{IU} / \mathrm{mL}$ ) may offer an explanation for why three individuals developed worsening of Ishak scores in our study. Last but not least, the presence of a hepatic repair complex ${ }^{26}$ among patients couldn't be provided to assess fibrosis regression, although pairing of the 7-stage Ishak fibrosis scoring and quantitative collagen parameter measurement was carried out in the current study. One multi-center randomized controlled clinical trial (NCT02241590) with large samples and longer following-up is currently being proposed based on our preliminary data from the small-sample investigator-initiated pilot trial.

In conclusion, in $\mathrm{CHB}$ patients with significant fibrosis/ cirrhosis, the combination therapy of FZHY plus ETV treatment for 48 weeks resulted in a higher rate of necroinflammatory improvement and fibrosis regression than that achieved with ETV alone. Parallel changes detected by quantitative collagen parameter measurement were consistent with this effect of FZHY. These findings provide convincing evidence to support the use of FZHY as an effective adjuvant for HBV-related significant fibrosis/cirrhosis with antiviral therapy.

\section{Acknowledgments}

We acknowledge Fang Yin, Jing-Jing Wang, and Qing-Shan Zheng for statistical assistance.

\section{Funding}

This work was supported by the Shanghai Three-Year Plan of the Key Subjects Construction in Public Health-Infectious Diseases and Pathogenic Microorganism (15GWZK0102), the Shanghai Three-Year Plan of the Clinical Skills and Innovations (16CR1002A), the Shanghai Municipal Key Clinical Specialty (shslczdzk01103), and Key Projects in the National Science and Technology Pillar Program during the Thirteen Five-year Plan Period (2017ZX10203202, 2017ZX10203201-008, 2017ZX10202202-005-004, 2018ZX09201016-003-001).

\section{Conflict of interest}

The authors have no conflict of interests related to this publication.

\section{Author contributions}

Designed the trial (QX and LMX), performed the trial (HLG, CQZ, WJW, HTG, WC, and QG), performed analysis using dual-photon microscopy (YW), provided trial input, interpreted the data and approved the manuscript (HLG, CQZ, YW, HTG, WJW, WC, QG, SSB, LMX and QX), wrote the manuscript and prepared the figures and tables with input (HLG, QX, and SSB).

\section{References}

[1] Pinzani M, Rosselli M, Zuckermann M. Liver cirrhosis. Best Pract Res Clin Gastroenterol 2011;25:281-290. doi: 10.1016/j.bpg.2011.02.009.

[2] Brown A, Goodman Z. Hepatitis B-associated fibrosis and fibrosis/cirrhosis regression with nucleoside and nucleotide analogs. Expert Rev Gastroenterol Hepatol 2012;6:187-198. doi: 10.1586/egh.12.4.

[3] Sun Y, Zhou J, Wang L, Wu X, Chen Y, Piao H, et al. New classification of liver biopsy assessment for fibrosis in chronic hepatitis $\mathrm{B}$ patients before and after treatment. Hepatology 2017;65:1438-1450. doi: 10.1002/hep.29009.

[4] Koyama Y, Xu J, Liu X, Brenner DA. New developments on the treatment of liver fibrosis. Dig Dis 2016;34:589-596. doi: 10.1159/000445269.

[5] Zhao CQ, Zhou Y, Ping J, Xu LM. Traditional Chinese medicine for treatment of liver diseases: progress, challenges and opportunities. J Integr Med 2014; 12:401-408. doi: 10.1016/S2095-4964(14)60039-X.

[6] Wang T, Zhou $X$, Liu H, Wang J, Zhang P, Zhu Y, et al. Fuzheng Huayu capsule as an adjuvant treatment for HBV-related cirrhosis: A systematic review and meta-analysis. Phytother Res 2018;32:757-768. doi: 10.1002/ptr.6009.

[7] Chen J, Hu Y, Chen L, Liu W, Mu Y, Liu P. The effect and mechanisms of Fuzheng Huayu formula against chronic liver diseases. Biomed Pharmacother 2019;114:108846. doi: 10.1016/j.biopha.2019.108846.

[8] Zhang L, Schuppan D. Traditional Chinese Medicine (TCM) for fibrotic liver disease: hope and hype. J Hepatol 2014;61:166-168. doi: 10.1016/j.jhep. 2014.03.009. 


\section{Gui H.L. et al: FZHY and ETV in CHB with significant fibrosis}

[9] Cao Z, Li Z, Wang H, Liu Y, Xu Y, Mo R, et al. Algorithm of Golgi protein 73 and liver stiffness accurately diagnoses significant fibrosis in chronic HBV infection. Liver Int 2017;37:1612-1621. doi: 10.1111/liv.13536.

[10] Gui HL, Wang $\mathrm{H}$, Yang YH, Wu YW, Zhou HJ, Guo SM, et al. Significant histopathology in Chinese chronic hepatitis B patients with persistently highnormal alanine aminotransferase. J Viral Hepat 2010;17 Suppl 1:44-50. doi: $10.1111 / j .1365-2893.2010 .01270 . x$.

[11] Goodman ZD. Grading and staging systems for inflammation and fibrosis in chronic liver diseases. J Hepatol 2007;47:598-607. doi: 10.1016/j.jhep. 2007.07.006

[12] Wang $Y$, Liang X, Yang J, Wang H, Tan D, Chen S, et al. Improved performance of quantitative collagen parameters versus standard histology in longitudinal assessment of nonadvanced liver fibrosis for chronic hepatitis B. J Viral Hepat 2018;25:598-607. doi: 10.1111/jvh.12835.

[13] Sun Y, Zhou J, Wu X, Chen Y, Piao H, Lu L, et al. Quantitative assessment of liver fibrosis (qFibrosis) reveals precise outcomes in Ishak "stable" patients on anti-HBV therapy. Sci Rep 2018;8:2989. doi: 10.1038/s41598-018-21179-2.

[14] Liu P, Hu YY, Liu C, Xu LM, Liu CH, Sun KW, et al. Multicenter clinical study on Fuzhenghuayu capsule against liver fibrosis due to chronic hepatitis $\mathrm{B}$. World J Gastroenterol 2005;11:2892-2899. doi: 10.3748/wjg.v11.i19.2892.

[15] Liu P, Liu C, Xu LM, Hu YY, Xue HM, Liu CH, et al. Effects of Fuzheng Huayu 319 recipe on liver fibrosis in chronic hepatitis B. World J Gastroenterol 1998; 4:348-353. doi: 10.3748/wjg.v4.i4.348.

[16] Almpanis Z, Demonakou M, Tiniakos D. Evaluation of liver fibrosis: "Something old, something new...". Ann Gastroenterol 2016;29:445-453. doi: 10. 20524/aog.2016.0046.

[17] Bedossa P, Carrat F. Liver biopsy: the best, not the gold standard. J Hepatol 2009;50:1-3. doi: 10.1016/j.jhep.2008.10.014.

[18] Xu S, Wang Y, Tai DCS, Wang S, Cheng CL, Peng Q, et al. qFibrosis: a fullyquantitative innovative method incorporating histological features to facilitate accurate fibrosis scoring in animal model and chronic hepatitis B patients. J Hepatol 2014;61:260-269. doi: 10.1016/j.jhep.2014.02.015

[19] Labenz C, Huber Y, Kalliga E, Nagel M, Ruckes C, Straub BK, et al. Predictor of advanced fibrosis in non-cirrhotic non-alcoholic fatty liver disease in Germany. Aliment Pharmacol Ther 2018;48:1109-1116. doi: 10.1111/apt. 14976.

[20] Lai CL, Shouval D, Lok AS, Chang TT, Cheinquer H, Goodman Z, et al. Entecavir versus lamivudine for patients with $\mathrm{HBeAg}$-negative chronic hepatitis B. N Engl J Med 2006;354:1011-1020. doi: 10.1056/NEJMoa051287.

[21] Chang TT, Gish RG, de Man R, Gadano A, Sollano J, Chao YC, et al. A comparison of entecavir and lamivudine for $\mathrm{HBeAg}$-positive chronic hepatitis $\mathrm{B}$. $\mathrm{N}$ Engl J Med 2006;354:1001-1010. doi: 10.1056/NEJMoa051285.

[22] Frenzel C, Teschke R. Herbal hepatotoxicity: clinical characteristics and listing compilation. Int J Mol Sci 2016;17:588. doi: 10.3390/ijms17050588.

[23] Jing ], Teschke R. Traditional Chinese medicine and herb-induced liver injury: Comparison with drug-induced liver injury. J Clin Transl Hepatol 2018;6:5768. doi: 10.14218/JCTH.2017.00033.

[24] Xing $X$, Chen $S$, Li L, Cao Y, Chen L, Wang X, et al. The active components of Fuzheng Huayu formula and their potential mechanism of action in inhibiting the hepatic stellate cells viability - A network pharmacology and transcriptomics approach. Front Pharmacol 2018;9:525. doi: 10.3389/fphar.2018. 00525 .

[25] Sun Y, Wu X, Zhou J, Meng T, Wang B, Chen S, et al. Persistent low level of hepatitis $\mathrm{B}$ virus promotes fibrosis progression during therapy. Clin Gastroenterol Hepatol 2020. doi: 10.1016/j.cgh.2020.03.001

[26] Hytiroglou P, Theise ND. Regression of human cirrhosis: an update, 18 years after the pioneering article by Wanless et al. Virchows Arch 2018;473:1522. doi: $10.1007 / \mathrm{s} 00428-018-2340-2$ 